\title{
Maternal High-fat Diet Leads to Persistent Synaptic Instability in Mouse Offspring via Oxidative Stress during Lactation
}

\section{Author names and affiliations}

Yusuke Hatanaka ${ }^{1,2,3 *}$, Keiji Wada ${ }^{1,2}$, Tomohiro Kabuta ${ }^{1, *}$

${ }^{1}$ Department of Degenerative Neurological Diseases, National Institute of Neuroscience, National Center of Neurology and Psychiatry, 4-1-1 Ogawa-Higashi, Kodaira, Tokyo 187-8502, Japan

${ }^{2}$ CREST, JST, 4-1-8 Honcho, Kawaguchi, Saitama 332-0012, Japan

${ }^{3}$ Department of of Neurology, Graduate School of Medicine, Kyoto University

54 Kawaharacho, Shogoin, Sakyo-ku, Kyoto CIty 606-8507, Japan

${ }^{*}$ Corresponding author

Tomohiro $\quad$ Kabuta $\quad$ (kabuta@ncnp.go.jp), $\quad$ Yusuke $\quad$ Hatanaka (hatanaka@kuhp.kyoto-u.ac.jp)

Phone: $+81-42-346-1715$ (ex. 5141 or 5840), Fax: $+81-42-346-1745$

Abbreviations: HFD, high-fat diet; ND, normal diet 


\section{Abstract}

2 Maternal obesity has negative effects on the neurodevelopment of the offspring. Pups

3 from high-fat diet (HFD)-fed mice exhibit peroxidized lipid accumulations in the brain

4 and behavioral impairments. However, the synaptic basis of maternal HFD-induced

5 brain dysfunction in offspring remains unclear. In the present study, we focused on the

6 dynamics and morphology of postsynaptic dendritic spines and filopodia in the

7 offspring of HFD-fed mouse dams, using in vivo two-photon imaging, chosen because

8 of the involvement of peripheral organs and non-neuronal cells in the abnormal

9 metabolic state. We observed instability of dendritic spines and filopodia in the cerebral

10 cortex of offspring from HFD-fed dams. Interestingly, the synaptic instability persisted

11 into adulthood with a lower spine density even when the offspring were fed with a

12 normal diet after weaning. HFD-fed offspring from HFD-fed dams showed a severe

13 disruption of dendritic spines. Synaptic instability and loss of spines were caused even

14 by HFD exposure exclusively during lactation. The treatment of ascorbic acid, an 15 antioxidant, during lactation ameliorated the synaptic impairments. These results

16 suggest that maternal obesity leads to persistent synaptic impairments in the offspring,

17 which may be associated with behavioral deficits in adulthood, and that these synaptic

18 deficits may be due to oxidative stress from peroxidized lipid accumulations during the

19 lactation period.

20

\section{Keywords}

22 Dendritic spine; in vivo two-photon imaging; high-fat diet; maternal environment; 23 lactation; antioxidant 


\section{1. Introduction}

25 Obesity is a worldwide health problem and a major contributor to the increased

26 incidence of coronary artery disease, hypertension and type 2 diabetes (Kopelman,

27 2000). Epidemiological studies have shown that maternal obesity has negative effects

28 on the neurodevelopment of children (Van Lieshout et al., 2011). Animal studies have

29 also demonstrated the effects of maternal obesity on the brain, as well as in the

30 peripheral organs of offspring (Williams et al., 2013). However, little is known about

31 the effects of maternal obesity on brain development in offspring. We previously

32 reported that mouse pups from dams fed with a high-fat diet (HFD) show peroxidized

33 lipid accumulations in many brain regions, impaired adult neurogenesis in the

34 hippocampus and deficits in spatial learning performance (Tozuka et al., 2010; Tozuka

35 et al., 2009). Other animal model studies have also demonstrated cognitive impairment

36 of offspring from HFD-fed dams (Rodriguez et al., 2012; Wu et al., 2013). However, the

37 synaptic basis for maternal HFD-induced brain dysfunction remains unclear. It is

38 possible that postsynaptic dendritic protrusions may be impaired in offspring born to

39 HFD-fed dams, because many behavioral deficits are associated with protrusion

40 impairment (Penzes et al., 2011). In our preceding study, peroxidized lipid

41 accumulations in the brain of the offspring from HFD-fed dams disappear when the

42 offspring are raised on a normal diet (ND) after weaning, whereas impaired progenitor

43 cell proliferation in the hippocampus and elevated locomotor activity persist into

44 adulthood (Tozuka et al., 2010; Tozuka et al., 2009). Thus, there may be reversible and

45 irreversible components of maternal HFD-induced brain impairment in offspring.

46 Accordingly, these lines of evidence raise the following questions about the impact of

47 maternal HFD on the synapses of the offspring: Are the synaptic impairments induced 
48 by maternal HFD reversible or persistent? When is the critical period for the synaptic 49 impairment in the offspring from HFD-fed dams? Is oxidative stress from peroxidized 50 lipids during the critical period a key factor in developing synaptic impairment?

51 In the present study, we analyzed the dynamics and morphology of dendritic 52 protrusions, classified into mature spines or immature filopodia, using in vivo 53 two-photon laser-scanning microscopy. We evaluated the synaptic deficits induced by 54 HFD in vivo because animals exposed to HFD show various pathologies in many 55 peripheral organs and non-neuronal cells in the brain (Bilbo and Tsang, 2010; Williams 56 et al., 2013). We used not only ND-fed offspring from HFD-fed dams but also HFD-fed 57 offspring from HFD-fed dams, to model western eating habits. Furthermore, in order to 58 elucidate the critical period when maternal HFD disrupts the synapses of the offspring, 59 we used ND-fed offspring from HFD-fed dams exclusively during lactation. We 60 examined the involvement of oxidative stress from peroxidized lipids during the critical 61 period by antioxidant-treatment. Our findings demonstrate that maternal HFD lead to 62 synaptic instability in the offspring mediated by oxidative stress during lactation, and 63 that the synaptic instability persisted into adulthood with developing loss of dendritic 64 spines. The present study is the first to examine the synaptic basis of brain dysfunction 65 in offspring born to HFD-fed dams. 


\section{2. Materials and Methods}

\section{$67 \quad 2.1 \quad$ Experimental animals}

68 Mice expressing yellow fluorescent protein (YFP) predominantly in layer (L) 5

69 pyramidal neurons (Thy1-YFP H-line) were purchased from the Jackson Laboratory and

70 backcrossed with C57BL/6J mice. Mice were housed four per cage under controlled

71 temperature $\left(25 \pm 1^{\circ} \mathrm{C}\right)$ and lighting $(12 \mathrm{~h}$ light/dark cycle), and were provided with

72 food and water ad libitum. The procedure of maternal HFD feeding and the

73 macronutrient composition and caloric distribution in the ND and HFD have been

74 described previously (Tozuka et al., 2009). Briefly, heterozygous Thy1-YFP mice were

75 fed ND (CE-2, CLEA Japan) or HFD (HFD-32, CLEA Japan) (Oike et al., 2005) for 6

76 weeks until mating. The same diet was maintained during pregnancy and for 15 days of

77 lactation. From lactational day 16, when pups first start to eat a solid food, pups were

78 assigned as follows: those born to HFD-fed dams but consuming ND (HFD/ND), those

79 from ND-fed dams but consuming HFD (ND/HFD), those from HFD-fed dams and

80 consuming HFD (HFD/HFD), and those from ND-fed dams and consuming ND

81 (ND/ND). Pups born to HFD-fed dams exclusively during lactation and consuming ND

82 were assigned to $\mathrm{HFD}_{\text {lad }}$ ND group. Pups from HFD-fed dams treated with $1 \mathrm{~g} / \mathrm{L}$

$83 L$-ascorbic acid (Vitamin C; Nacalai Tesque, Japan) in drinking water during lactation

84 and consuming ND were assigned to $\mathrm{HFD}_{\mathrm{AA}} / \mathrm{ND}$ group. The fluid intake of mice

85 received normal drinking water or drinking water supplemented with

86 ascorbic acid is approximately $10 \mathrm{~mL} /$ day (Abdel-Wahab et al., 2002). On the

87 day of parturition (day 0 ), litters were culled to 6-8 offspring per mother. Pups were

88 kept with their dams until weaning on day 22. Only male offspring heterozygous for

89 YFP were used for all analyses. To avoid autoxidation of the HFD, diets were stored at 
$90-80^{\circ} \mathrm{C}$ until use, and food was renewed every $2 \mathrm{~d}$. Animal procedures were in strict

91 accordance with the guidelines of the National Institute of Neuroscience, National

92 Center of Neurology and Psychiatry (Japan).

93

\section{$94 \quad 2.2 \quad$ Surgical procedure for in vivo imaging}

95 We used the thinned-skull cranial window technique (Yang et al., 2010) because it is

96 less invasive than the open-skull method (Xu et al., 2007). Male pups expressing YFP

97 were deeply anesthetized with urethane $(2 \mathrm{mg} / \mathrm{g}$ body weight, i.p.). Body temperature

98 was maintained at $37^{\circ} \mathrm{C}$ with a heating pad during the surgery and following imaging.

99 After the scalp incision, both eyes were lubricated with eye ointment, and the primary

100 somatosensory area $(1.1 \mathrm{~mm}$ posterior to bregma and $3.4 \mathrm{~mm}$ lateral from midline $)$ was

101 identified with stereotactic coordinates. A small metal plate with a round hole was glued

102 onto the skull with cyanoacrylate glue and acrylic resin dental cement (UNIFAST; GC,

103 Japan), and mice were fixed to a custom-made skull immobilization stage via the metal

104 plate. The skull above the imaging area, located in the center of the hole in the metal

105 plate, was thinned to a thickness of approximately $20 \mu \mathrm{m}$ with a high-speed microdrill

106 (UG23A/UC210C; Urawa, Japan) and microsurgical blade (USM-6400; Sable

107 Industries, Vista, CA, USA). The hole in the metal plate was filled with artificial

108 cerebrospinal fluid during surgery and imaging.

109

$1102.3 \quad$ In vivo transcranial two-photon imaging

111 Thy1-YFP pups were imaged under anesthesia using two-photon laser scanning 112 microscopy (FV1000-MPE; Olympus, Japan) with a water-immersion objective lens $113(25 \times$, NA 1.05$)$ at $8 \times$ digital zoom yielding high-magnification images suitable for 
114 quantification of dendritic spines. A Ti-sapphire laser (MaiTai HP DeepSee-OL;

115 Spectra-Physics, Mountain View, CA, USA) was tuned to $950 \mathrm{~nm}$. Laser intensity was

116 kept in the range of $10-30 \mathrm{~mW}$ at the focus to minimize phototoxicity. Image-stacks

$117(512 \times 512$ pixels; $0.124 \mu \mathrm{m} /$ pixels; $0.75 \mu \mathrm{m}$ z-step $)$ were taken at approximately $70 \mu \mathrm{m}$

118 below the pial surface, where L1 dendrites of L5 pyramidal neurons are located.

119 Dendritic segments were imaged for each experiment at time 0, and then the same

120 dendritic segments were imaged again after an interval of $1 \mathrm{~h}$. All images were acquired

121 carefully to approximate similar fluorescence levels across imaged regions within each

122 experiment and across imaging sessions in different animals. The animals were

123 sacrificed immediately after imaging sessions.

124

$125 \quad 2.4 \quad$ Image analysis

126 The turnover rate, density, head width, and neck length of dendritic protrusions were

127 analyzed with Neurolucida (MicroBrightField, Williston, VT, USA) from

128 three-dimensional two-photon z-stacks. Morphometric analysis of dendritic protrusions

129 was in accordance with a previous report (Grutzendler et al., 2002). Dendritic

130 protrusions were classified into two groups, spines and filopodia, and

131 formation/elimination rate were analyzed in each group. Filopodia were identified as

132 long, thin structures (ratio of head width to neck width $<1.2: 1$; ratio of length to neck

133 width $>3: 1$ ). The remaining protrusions were classified as spines. These protrusions

134 were considered the same between two views on the basis of their spatial relationship to

135 adjacent landmarks and their relative position to immediately adjacent protrusions.

136 Protrusions were considered different if they were $>0.7 \mu \mathrm{m}$ from their expected

137 positions based on the first view. The formation and elimination rates for the protrusions 
138 were defined as the fraction of protrusions that appeared and disappeared, respectively, 139 between two successive frames, relative to the total protrusion number. Protrusion 140 turnover rate was defined as the sum of the protrusions formed and eliminated divided

141 by twice the total number of protrusions. Data were collected from 10-21 dendrites and 142 approximately 1,000 protrusions in four to seven mice.

143

$144 \quad 2.5 \quad$ Statistical analysis

145 To determine statistical significance, we used Student's $t$ test, a one-way ANOVA 146 followed by Tukey's multiple-comparison test, a two-way ANOVA followed by 147 Bonferroni's multiple-comparison test, or a Kolmogorov-Smirnov test. All statistical 148 analyses were performed with GraphPad Prism (GraphPad Software Inc, La Jolla, CA, 149 USA). All data are presented as mean \pm SEM, where $n=$ number of dendrites. 


\section{Results}

$1513.1 \quad$ Maternal HFD leads to instability of dendritic spines and filopodia in ND-fed

152 adult offspring

153 In order to evaluate the effects of maternal HFD on the synapses in adult offspring

154 consuming ND, we performed in vivo two-photon imaging in L1 dendrites in the

155 primary somatosensory cortex of 10-week-old ND-fed offspring of ND- or HFD-fed

156 dams (ND/ND or HFD/ND) (Figure 1a). We analyzed the formation and elimination of

157 dendritic spines and filopodia within $1 \mathrm{~h}$ (Figure 1b). Compared with ND/ND mice, the

158 elimination rate of spines in 10-week-old HFD/ND mice was significantly greater, while

159 the formation rate was not significantly different (Figure 1c; formation rate: ND/ND:

$1602.9 \pm 0.9 \%, n=11 ; \mathrm{HFD} / \mathrm{ND}: 5.3 \pm 0.7 \%, n=21 ; p=0.0516$; elimination rate: ND/ND:

$1611.1 \pm 0.4 \%, n=11 ; \mathrm{HFD} / \mathrm{ND}: 7 \pm 1 \%, n=21 ; p=0.0025 ;$ unpaired $t$ test). Both

162 formation and elimination rate of filopodia were greater in HFD/ND mice (Figure 1d;

163 formation rate: ND/ND: $6 \pm 3 \%, n=11$; HFD/ND: $28 \pm 4 \%, n=21 ; p=0.0020$;

164 elimination rate: ND/ND: $2 \pm 2 \%, n=11$; HFD/ND: $32 \pm 6 \%, n=21 ; p=0.0035$;

165 unpaired $t$ test). These data show that maternal HFD causes synaptic instability in adult

166 offspring even though the offspring are fed ND after weaning.

167

$1683.2 \quad$ Offspring exposed to maternal HFD develop synaptic instability and loss of

169 dendritic spines during synaptic maturation

170 To reveal how the synapses are impaired after weaning, we investigated the effects of

171 maternal HFD on synaptic development during juvenile and adulthood in ND/ND and

$172 \mathrm{HFD} / \mathrm{ND}$ mice at 4, 6, 8 and 10 weeks of age. Because normal dendritic spines are 
173 gradually stabilized across neuronal circuitry development (Grutzendler et al., 2002),

174 we analyzed the turnover rate of spines and filopodia at each age during synaptic

175 development. In HFD/ND mice, spine turnover rate was significantly greater than in

176 ND/ND mice throughout synaptic development (Figure 2a; 4 wks: $p<0.0001 ; 6$ wks: $p$

$177<0.0001 ; 8$ wks: $p<0.0001 ; 10$ wks: $p=0.0148$; two-way ANOVA followed by

178 Bonferroni's test), and filopodium turnover rate was greater at 6,8 , and 10 weeks of age

179 in HFD/ND mice (Figure 2b; 4 wks: $p=0.3612 ; 6$ wks: $p<0.0001 ; 8$ wks: $p<0.0001$;

18010 wks: $p<0.0001$; two-way ANOVA followed by Bonferroni's test). No significant

181 difference was observed in filopodium turnover rate at 4 weeks of age. This may be due

182 to the high turnover rate of filopodia at this immature stage of synaptic development

183 even in the control group. Note that, in ND/ND mice, turnover rates of spines and

184 filopodia were gradually decreased with normal maturation. Next, we analyzed the

185 density of dendritic spines and filopodia during synaptic development. In 4-week-old

186 HFD/ND mice, only filopodium density was significantly greater than that in ND/ND

187 mice, while the densities of total protrusions and spines were not different between

188 groups (Figure 2c; protrusions: ND/ND: $0.38 \pm 0.02$ protrusions $/ \mu \mathrm{m}, n=13$; HFD/ND:

$1890.40 \pm 0.03$ protrusions $/ \mu \mathrm{m}, n=18 ; p=0.6432$; spines: ND/ND: $0.31 \pm 0.02$ spines $/ \mu \mathrm{m}$,

$190 n=13 ; \mathrm{HFD} / \mathrm{ND}: 0.29 \pm 0.02$ spines $/ \mu \mathrm{m}, n=18 ; p=0.3919 ;$ filopodia: ND/ND: 0.070

$191 \pm 0.006$ filopodia/ $\mu \mathrm{m}, n=13 ; \mathrm{HFD} / \mathrm{ND}: 0.11 \pm 0.01$ filopodia/ $\mu \mathrm{m}, n=18 ; p=0.0243$;

192 unpaired $t$ test). At 6 weeks of age, there were no significant differences in protrusion, 193 spine, or filopodium density between ND/ND and HFD/ND mice (Figure 2c;

194 protrusions: ND/ND: $0.39 \pm 0.02$ protrusions/ $\mu \mathrm{m}, n=12 ; \mathrm{HFD} / \mathrm{ND}: 0.38 \pm 0.02$

195 protrusions $/ \mu \mathrm{m}, n=18 ; p=0.8050$; spines: ND/ND: $0.33 \pm 0.02$ spines $/ \mu \mathrm{m}, n=12$;

196 HFD/ND: $0.31 \pm 0.02$ spines/ $\mu \mathrm{m}, n=18 ; p=0.5866$; filopodia: ND/ND: $0.06 \pm 0.01$ 
197 filopodia/ $\mu \mathrm{m}, n=12 ; \mathrm{HFD} / \mathrm{ND}: 0.065 \pm 0.007$ filopodia/ $\mu \mathrm{m}, n=18 ; p=0.5995$;

198 unpaired $t$ test). In 8-week-old mice, spine density was significantly lower whereas

199 filopodium density was greater in HFD/ND mice than in ND/ND mice; however, total

200 protrusion density was not significantly different (Figure 2c; protrusions: ND/ND: 0.39

$201 \pm 0.01$ protrusions $/ \mu \mathrm{m}, n=15 ; \mathrm{HFD} / \mathrm{ND}: 0.36 \pm 0.02$ protrusions $/ \mu \mathrm{m}, n=20 ; p=$

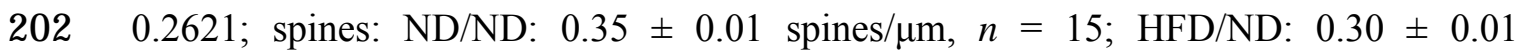

203 spines $/ \mu \mathrm{m}, n=20 ; p=0.0194$; filopodia: ND/ND: $0.039 \pm 0.004$ filopodia/ $\mu \mathrm{m}, n=15$;

204 HFD/ND: $0.061 \pm 0.008$ filopodia/ $\mu \mathrm{m}, n=20 ; p=0.0340$; unpaired $t$ test). In

205 10-week-old HFD/ND mice, spine density was considerably lower than in ND/ND mice,

206 while filopodium density was not different; therefore, total protrusion density was

207 significantly lower (Figure 2c; protrusions: ND/ND: $0.39 \pm 0.02$ protrusions $/ \mu \mathrm{m}, n=11$;

$208 \mathrm{HFD} / \mathrm{ND}: 0.32 \pm 0.01$ protrusions $/ \mu \mathrm{m}, n=21 ; p=0.0208$; spines: ND/ND: $0.35 \pm 0.01$

209 spines $/ \mu \mathrm{m}, n=11 ; \mathrm{HFD} / \mathrm{ND}: 0.28 \pm 0.01$ spines $/ \mu \mathrm{m}, n=21 ; p=0.0005$; filopodia:

210 ND/ND: $0.03 \pm 0.02$ filopodia/ $\mu \mathrm{m}, n=11 ; \mathrm{HFD} / \mathrm{ND}: 0.047 \pm 0.004$ filopodia/ $\mu \mathrm{m}, n=$

$21121 ; p=0.3034$; unpaired $t$ test). Note that, in normal synaptic development, the ratio of

212 filopodia to spines is gradually decreased, but HFD/ND mice showed persistent higher

213 ratio of filopodia to spines. These results indicate that the normal development of

214 dendritic spines and filopodia in HFD/ND mice is impaired by maternal HFD, and the

215 impairment of synaptic dynamics and morphology persists into adulthood.

216

$217 \quad 3.3 \quad H F D$-fed offspring from HFD-fed dams show a severe loss of dendritic spines

218 Several reports indicate that HFD exposure in adulthood without maternal dietary effect

219 causes serious damage to cognitive function, synaptic plasticity and spine density

220 (Stranahan et al., 2008; Valladolid-Acebes et al., 2013; Wu et al., 2004). To evaluate the 
221 damage of a western-style eating habit, in which both mothers and children are exposed 222 to HFD throughout life, we investigated dynamics and morphology of dendritic spines 223 and filopodia in maternal HFD-exposed offspring given HFD after weaning. We 224 performed in vivo imaging in L1 dendrites of HFD-fed 8-week-old pups from HFD-fed 225 dams (HFD/HFD), and compared the results with age-matched ND/ND, HFD/ND and 226 ND/HFD groups (Figure 3a). ND/HFD group is a model of HFD exposure exclusively 227 after weaning and is assigned to a control group. We analyzed the formation and 228 elimination of dendritic spines and filopodia within $1 \mathrm{~h}$ (Figure $3 \mathrm{~b}$ ). Both formation and 229 elimination rates of spines in HFD/HFD mice were significantly greater than those in $230 \mathrm{ND} / \mathrm{ND}$ mice (Figure 3c; formation rate: ND/ND: $1.7 \pm 0.4 \%, n=15$; HFD/HFD: $6 \pm$ $2311 \%, n=12 ; p=0.0021$; elimination rate: ND/ND: $1.4 \pm 0.5 \%, n=15 ;$ HFD/HFD: $11 \pm$ $2322 \%, n=12 ; p=0.0001$; one-way ANOVA followed by Tukey's test). There were no 233 significant differences in spine formation and elimination rate among HFD/ND, 234 ND/HFD and HFD/HFD mice (Figure 3c; formation rate: HFD/ND: $5.6 \pm 0.8 \%, n=20$; 235 ND/HFD: $7.2 \pm 0.9 \%, n=16$; HFD/HFD: $6 \pm 1 \%, n=12$; elimination rate: HFD/ND: $23610 \pm 1 \%, n=20$; ND/HFD: $12 \pm 1 \%, n=16$; HFD/HFD: $11 \pm 2 \%, n=12$; one-way 237 ANOVA followed by Tukey's test). Formation and elimination rates of filopodia were 238 significantly greater in HFD/HFD mice than those in ND/ND mice (Figure 3d; 239 formation rate: ND/ND: $5 \pm 2 \%, n=15$; HFD/HFD: $25 \pm 6 \%, n=12 ; p=0.0003$; 240 elimination rate: ND/ND: $9 \pm 3 \%, n=15$; HFD/HFD: $28 \pm 5 \%, n=12 ; p=0.0009$; 241 one-way ANOVA followed by Tukey's test). There were no significant differences in 242 rate of filopodium formation or elimination among HFD/ND, ND/HFD and HFD/HFD 243 mice (Figure 3d; formation rate: HFD/ND: $40 \pm 4 \%, n=20$; ND/HFD: $46 \pm 5 \%, n=16$; 244 HFD/HFD: $33 \pm 5 \%, n=12$; elimination rate: HFD/ND: $36 \pm 4 \%, n=20 ; \mathrm{ND} / \mathrm{HFD}: 38$ 
$245 \pm 6 \%, n=16$; HFD/HFD: $32 \pm 5 \%, n=12$; one-way ANOVA followed by Tukey's test).

246 Note that HFD feeding after weaning caused synaptic instability even without the effect

247 of maternal HFD. These data indicate that pre- and post-weaning HFD exposure leads

248 to synaptic instability in later life, respectively, and that they have no additive effects on

249 the dynamics of dendritic spines and filopodia. The density of dendritic spines in

250 HFD/HFD group was notably lower than any other group (Figure 3e; spines: ND/ND:

$2510.35 \pm 0.01$ spines $/ \mu \mathrm{m}, n=15 ; \mathrm{HFD} / \mathrm{ND}: 0.30 \pm 0.01$ spines $/ \mu \mathrm{m}, n=20 ; \mathrm{ND} / \mathrm{HFD}: 0.33$

$252 \pm 0.02$ spines/ $\mu \mathrm{m}, n=16 ; \mathrm{HFD} / \mathrm{HFD}: 0.23 \pm 0.01$ spines/ $\mu \mathrm{m}, n=12 ; p<0.0001$

253 (compared to ND/ND), $p=0.0105$ (compared to HFD/ND), $p=0.0004$ (compared to

254 ND/HFD); one-way ANOVA followed by Tukey's test). There were no significant

255 differences among all groups in filopodium density (Figure 3e; filopodia: ND/ND:

$2560.039 \pm 0.004$ filopodia/ $\mu \mathrm{m}, n=15 ; \mathrm{HFD} / \mathrm{ND}: 0.060 \pm 0.008$ filopodia/ $\mu \mathrm{m}, n=20$;

257 ND/HFD: $0.064 \pm 0.007$ filopodia/ $\mu \mathrm{m}, n=16$; HFD/HFD: $0.060 \pm 0.008$ filopodia/ $\mu \mathrm{m}$,

$258 n=12$; one-way ANOVA followed by Tukey's test). As a result, total protrusion density

259 in HFD/HFD group was lower than the others (Figure 3e; protrusions: ND/ND: $0.39 \pm$

2600.01 protrusions $/ \mu \mathrm{m}, n=15 ; \mathrm{HFD} / \mathrm{ND}: 0.36 \pm 0.02$ protrusions $/ \mu \mathrm{m}, n=20 ; \mathrm{ND} / \mathrm{HFD}$ :

$2610.40 \pm 0.03$ protrusions $/ \mu \mathrm{m}, n=16$; HFD/HFD: $0.29 \pm 0.01$ protrusions $/ \mu \mathrm{m}, n=12 ; p=$

2620.0055 (compared to ND/ND), $p=0.0429$ (compared to HFD/ND), $p=0.0030$

263 (compared to ND/HFD); one-way ANOVA followed by Tukey's test). Note that

264 significant loss of dendritic spines is displayed only in HFD/HFD group. Interestingly,

265 synaptic instability of HFD/HFD mice is equal to that of HFD/ND and ND/HFD mice

266 although spine density was significantly lower only in HFD/HFD mice.

267

$2683.4 \quad$ Synaptic instability and loss of dendritic spines are caused even by limited 
270 In order to elucidate the critical period when maternal HFD impairs the synaptic 271 dynamics and morphology in the mature offspring, we evaluated dynamics and 272 morphology of dendritic spines and filopodia in L1 dendrites of the offspring exposed to 273 maternal HFD exclusively during lactation ( $\mathrm{HFD}_{\mathrm{lac}} \mathrm{ND}$ ) (Figure $\left.4 a\right)$. We analyzed the 274 formation and elimination of dendritic spines and filopodia over $1 \mathrm{~h}$ in 8 -week-old $275 \mathrm{HFD}_{\text {lac }} / \mathrm{ND}$ mice in vivo (Figure $4 \mathrm{~b}$ ). Both formation and elimination rates of spines in $276 \mathrm{HFD}_{\text {lac }} / \mathrm{ND}$ mice were greater than those in ND/ND mice (Figure $4 \mathrm{c}$; formation rate: $277 \mathrm{ND} / \mathrm{ND}: 1.7 \pm 0.4 \%, n=15 ; \mathrm{HFD}_{\mathrm{lac}} \mathrm{ND}: 8 \pm 1 \%, n=15 ; p<0.0001$; elimination rate: $278 \mathrm{ND} / \mathrm{ND}: 1.4 \pm 0.5 \%, n=15 ; \mathrm{HFD}_{\mathrm{lad}} \mathrm{ND}: 11 \pm 2 \%, n=15 ; p<0.0001$; one-way 279 ANOVA followed by Tukey's test). Furthermore, $\mathrm{HFD}_{\mathrm{lac}}$ (ND mice showed greater 280 formation rate of spines than HFD/ND mice (Figure 4c; $p=0.0401$; one-way ANOVA 281 followed by Tukey's test). Formation and elimination rates of filopodia were 282 significantly greater in $\mathrm{HFD}_{\mathrm{lac}} \mathrm{ND}$ mice than those in ND/ND mice (Figure 4d; 283 formation rate: $\mathrm{ND} / \mathrm{ND}: 5 \pm 2 \%, n=15 ; \mathrm{HFD}_{\mathrm{lad}} / \mathrm{ND}: 47 \pm 5 \%, n=15 ; p<0.0001$; 284 elimination rate: ND/ND: $9 \pm 3 \%, n=15 ; \mathrm{HFD}_{\mathrm{lad}} \mathrm{ND}: 44 \pm 8 \%, n=15 ; p=0.0002$; 285 one-way ANOVA followed by Tukey's test). There were no significant differences in 286 rate of filopodium formation or elimination between $\mathrm{HFD} / \mathrm{ND}$ and $\mathrm{HFD}_{\text {lac }} / \mathrm{ND}$ mice 287 (Figure 4d). These data indicate that synaptic instability of the mature offspring is 288 triggered even by maternal HFD exposure exclusively during lactation. There were a 289 significant difference in spine density only between ND/ND and $\mathrm{HFD}_{\mathrm{lac}} / \mathrm{ND}$ group, and 290 spine density in $\mathrm{HFD}_{\text {lac }}$ /ND mice was lower than ND/ND mice (Figure 4e; spines: 291 ND/ND: $0.35 \pm 0.01$ spines/ $\mu \mathrm{m}, n=15 ; \mathrm{HFD}_{\mathrm{lac}} \mathrm{ND}: 0.29 \pm 0.02$ spines $/ \mu \mathrm{m}, n=15 ; p=$ 292 0.0273; one-way ANOVA followed by Tukey's test). There were no significant 
293 differences among ND/ND, HFD/ND and $\mathrm{HFD}_{\mathrm{lac}} / \mathrm{ND}$ in filopodium density (Figure 4e;

294 filopodia: ND/ND: $0.039 \pm 0.004$ filopodia/ $\mu \mathrm{m}, n=15 ; \mathrm{HFD} / \mathrm{ND}: 0.060 \pm 0.008$

295 filopodia/ $\mu \mathrm{m}, n=20 ; \mathrm{HFD}_{\text {lac }} / \mathrm{ND}: 0.06 \pm 0.01$ filopodia/ $\mu \mathrm{m}, n=15$; one-way ANOVA

296 followed by Tukey's test) and total protrusion density (Figure 4e; protrusions: ND/ND:

$2970.39 \pm 0.01$ protrusions $/ \mu \mathrm{m}, n=15 ; \mathrm{HFD} / \mathrm{ND}: 0.36 \pm 0.02$ protrusions $/ \mu \mathrm{m}, n=20$

$298 \mathrm{HFD}_{\text {lac }}$ ND: $0.35 \pm 0.02$ protrusions/ $\mu \mathrm{m}, n=15$; one-way ANOVA followed by Tukey's

299 test). It is interesting that the synaptic dynamics and morphology are more disrupted in $300 \mathrm{HFD}_{\text {lac }} / \mathrm{ND}$ mice than in HFD/ND mice.

301

$302 \quad 3.5 \quad$ Antioxidant-treatment during lactation ameliorates the synaptic impairment 303 in the offspring from HFD-fed dams

304 Several lines of evidence show that HFD-induced cognitive impairment is due to 305 oxidative stress in the brain (Souza et al., 2007; Zhang et al., 2005). Furthermore, we 306 have previously demonstrated that maternal HFD leads to peroxidized lipid 307 accumulations in the brain of the offspring during early postnatal development (Tozuka 308 et al., 2009). An index of lipid peroxidation, 2-thiobarbituric acid reactive substances, is 309 increased approximately 1.5 -fold by maternal HFD in our experimental conditions 310 (Tozuka et al., 2009). Peroxidized lipid accumulations in the brain are significantly 311 decreased by the treatment of ascorbic acid, an antioxidant, in drinking water (Harrison 312 et al., 2010). According to those reports and the present results, we hypothesized that 313 maternal HFD-induced oxidative stress during lactation, when synapses are immature, 314 causes persistent impairments in synaptic development of the offspring, even if they are 315 fed with ND after weaning. In order to elucidate the involvement of lipid-mediated 316 oxidative stress during lactation in the synaptic impairments of the offspring, we treated 
317 the HFD-fed dams with ascorbic acid, exclusively during lactation, and performed in 318 vivo imaging in L1 dendrites of the 10-week-old offspring ( $\left.\mathrm{HFD}_{\mathrm{AA}} / \mathrm{ND}\right)$ (Figure 5a). $319 \mathrm{ND}_{\mathrm{AA}} / \mathrm{ND}$ mice are offspring from ND-fed and ascorbic acid-treated dams, and used as 320 a control group. We analyzed the formation and elimination of dendritic spines and 321 filopodia within $1 \mathrm{~h}$ (Figure 5b). Both formation and elimination rates of spines in $322 \mathrm{HFD}_{\mathrm{AA}} / \mathrm{ND}$ mice were significantly lower than those in HFD/ND mice (Figure $5 \mathrm{c}$; 323 formation rate: $\mathrm{HFD} / \mathrm{ND}: 5.3 \pm 0.7 \%, n=21 ; \mathrm{HFD}_{\mathrm{AA}} / \mathrm{ND}: 1.7 \pm 0.5 \%, n=10 ; p=$ 3240.0063 ; elimination rate: $\mathrm{HFD} / \mathrm{ND}: 7 \pm 1 \%, n=21 ; \mathrm{HFD}_{\mathrm{AA}} / \mathrm{ND}: 1.7 \pm 0.4 \%, n=10 ; p=$ 325 0.0004; one-way ANOVA followed by Tukey's test). There were no significant 326 differences in spine formation and elimination rate among $\mathrm{ND} / \mathrm{ND}, \mathrm{ND}_{\mathrm{AA}} / \mathrm{ND}$ and $327 \mathrm{HFD}_{\mathrm{AA}} / \mathrm{ND}$ mice (Figure $5 \mathrm{c}$; formation rate: ND/ND: $2.9 \pm 0.9 \%, n=11 ; \mathrm{ND}_{\mathrm{AA}} / \mathrm{ND}$ : $3282.0 \pm 0.7 \%, n=10 ; \mathrm{HFD}_{\mathrm{AA}} / \mathrm{ND}: 1.7 \pm 0.5 \%, n=10 ;$ elimination rate: $\mathrm{ND} / \mathrm{ND}: 1.1 \pm$ $3290.4 \%, n=11 ; \mathrm{ND}_{\mathrm{AA}} / \mathrm{ND}: 0.9 \pm 0.3 \%, n=10 ; \mathrm{HFD}_{\mathrm{AA}} / \mathrm{ND}: 1.7 \pm 0.4 \%, n=10$; one-way 330 ANOVA followed by Tukey's test). Although only elimination rate of filopodia was 331 significantly decreased between $\mathrm{HFD} / \mathrm{ND}$ and $\mathrm{HFD}_{\mathrm{AA}} / \mathrm{ND}$ mice (Figure $5 \mathrm{~d}$; formation 332 rate: $\mathrm{HFD} / \mathrm{ND}: 28 \pm 4 \%, n=21 ; \mathrm{HFD}_{\mathrm{AA}} / \mathrm{ND}: 17 \pm 2 \%, n=10 ; p=0.2140$; elimination 333 rate: $\mathrm{HFD} / \mathrm{ND}: 32 \pm 6 \%, n=21 ; \mathrm{HFD}_{\mathrm{AA}} / \mathrm{ND}: 10 \pm 3 \%, n=10 ; p=0.0315$; one-way 334 ANOVA followed by Tukey's test), there were no significant differences in filopodium 335 formation and elimination rate among $\mathrm{ND} / \mathrm{ND}, \mathrm{ND}_{\mathrm{AA}} / \mathrm{ND}$ and $\mathrm{HFD}_{\mathrm{AA}} / \mathrm{ND}$ mice (Figure $3365 \mathrm{~d}$; formation rate: $\mathrm{ND} / \mathrm{ND}: 6 \pm 3 \%, n=11 ; \mathrm{ND}_{\mathrm{AA}} / \mathrm{ND}: 16 \pm 3 \%, n=10 ; \mathrm{HFD}_{\mathrm{AA}} / \mathrm{ND}$ : $33717 \pm 2 \%, n=10 ;$ elimination rate: ND/ND: $2 \pm 2 \%, n=11 ; \mathrm{ND}_{\mathrm{AA}} / \mathrm{ND}: 12 \pm 4 \%, n=10$; $338 \mathrm{HFD}_{\mathrm{AA}} / \mathrm{ND}: 10 \pm 3 \%, n=10$; one-way ANOVA followed by Tukey's test). In spine 339 density, although there were no significant differences between $\mathrm{HFD}_{\mathrm{AA}} / \mathrm{ND}$ and 340 HFD/ND mice, there were also no significant differences among ND/ND, $\mathrm{ND}_{\mathrm{AA}} / \mathrm{ND}$ 
341 and $\mathrm{HFD}_{\mathrm{AA}} / \mathrm{ND}$ mice (Figure 5e; spines: ND/ND: $0.35 \pm 0.01$ spines $/ \mu \mathrm{m}, n=11$;

$342 \mathrm{HFD} / \mathrm{ND}: 0.28 \pm 0.01$ spines $/ \mu \mathrm{m}, n=21 ; \mathrm{ND}_{\mathrm{AA}} / \mathrm{ND}: 0.37 \pm 0.02$ spines $/ \mu \mathrm{m}, n=10$;

$343 \mathrm{HFD}_{\mathrm{AA}} / \mathrm{ND}: 0.32 \pm 0.02$ spines$/ \mu \mathrm{m}, n=10$; one-way ANOVA followed by Tukey's test).

344 There were no significant differences among all groups in filopodium density (Figure

345 5e; filopodia: ND/ND: $0.03 \pm 0.02$ filopodia/ $\mu \mathrm{m}, n=11 ; \mathrm{HFD} / \mathrm{ND}: 0.047 \pm 0.004$

346 filopodia/ $\mu \mathrm{m}, n=21 ; \mathrm{ND}_{\mathrm{AA}} / \mathrm{ND}: 0.044 \pm 0.007$ filopodia/ $\mu \mathrm{m}, n=10 ; \mathrm{HFD}_{\mathrm{AA}} / \mathrm{ND}$ :

$3470.045 \pm 0.006$ filopodia/ $\mu \mathrm{m}, n=10$; one-way ANOVA followed by Tukey's test). In

348 protrusion density, there were no significant differences between $\mathrm{HFD}_{\mathrm{AA}} / \mathrm{ND}$ and

$349 \mathrm{HFD} / \mathrm{ND}$ mice, and there were also no significant differences among ND/ND,

$350 \mathrm{ND}_{\mathrm{AA}} / \mathrm{ND}$ and $\mathrm{HFD}_{\mathrm{AA}} / \mathrm{ND}$ mice (Figure 5e; protrusions: ND/ND: $0.39 \pm 0.02$

351 protrusions $/ \mu \mathrm{m}, n=11$; HFD/ND: $0.32 \pm 0.01$ protrusions $/ \mu \mathrm{m}, n=21 ; \mathrm{ND}_{\mathrm{AA}} / \mathrm{ND}: 0.42$

$352 \pm 0.02$ protrusions $/ \mu \mathrm{m}, n=10 ; \mathrm{HFD}_{\mathrm{AA}} / \mathrm{ND}: 0.37 \pm 0.03$ protrusions $/ \mu \mathrm{m}, n=10$

353 one-way ANOVA followed by Tukey's test). These data indicate that ascorbic acid

354 treatment during lactation completely rescued the instability of dendritic spines, and 355 partially ameliorated the filopodium instability and spine density. 


\section{4. Discussion}

358 A typical diet in the developed world is an energy-rich diet containing high levels of fats

359 and sugars. Chronic consumption of this type of diet is associated with adverse effects

360 on health, and perinatal HFD exposure also causes similar effects on the offspring.

361 Many women are overweight or obese by the time they reach childbearing age (Huda et

362 al., 2010; Kanagalingam et al., 2005). Therefore, there is a great need to elucidate the

363 mechanisms by which maternal HFD can affect synaptic development and brain

364 function. In the present study, we found that maternal HFD led to instability of dendritic

365 spines and filopodia in the cerebral cortex of juvenile offspring even, when they were

366 fed with a ND after weaning. These negative effects persisted into adulthood with a

367 decline in dendritic spines. HFD-fed offspring from HFD-fed dams showed a severe

368 decline in dendritic spines, whereas synaptic instability in HFD/HFD mice was equal to

369 that in HFD/ND and ND/HFD mice. Synaptic instability and loss of spines in the

370 offspring exposed to maternal HFD exclusively during lactation were greater than or

371 equal to those in the offspring exposed to pre- and postnatal maternal HFD.

372 Antioxidant-treatment during lactation ameliorated the synaptic impairment in the

373 offspring born to HFD-fed dams. These results suggest that maternal obesity causes

374 sustained synaptic impairments in the offspring, which may be associated with brain

375 dysfunction in adulthood, and that these impairments may be due to oxidative stress

376 from peroxidized lipid accumulations during lactation. The perinatal metabolic milieu is

377 one of the most important factors that have an impact on neuronal circuitry development

378 and brain function in adulthood. This study provides the first evidence of the synaptic

379 basis of the brain dysfunction in the offspring from obese dams, using in vivo imaging

380 while maintaining the internal environment of the body. 
381 Animal models of synaptic development in children of obese mothers

382 In the present study, we used a well-established animal model of maternal obesity.

383 Dams were fed HFD 6 weeks prior to mating until weaning. The protocol is known to

384 replicate human pathology in the offspring, such as abnormal insulin and glucose

385 metabolism, energy balance, cardiovascular function and adiposity (Elahi et al., 2009;

386 Ferezou-Viala et al., 2007; Samuelsson et al., 2008; Tozuka et al., 2010; Tozuka et al.,

387 2009). We have previously demonstrated that the offspring from HFD-fed dams shows

388 increased body weight and blood glucose without any changes in food intake (Tozuka et

389 al., 2009). Other models of maternal obesity also demonstrate converging effects on the

390 brain and peripheral organs (Williams et al., 2013). We can compare the results of these

391 different model studies in the context of maternal obesity.

392 In order to evaluate the synapse pathologies of maternal obesity, we

393 performed in vivo two-photon imaging because there are large contributions from

394 peripheral organs and non-neuronal cells in the offspring from HFD-fed dams showing

395 abnormal metabolic homeostasis (Bilbo and Tsang, 2010; Williams et al., 2013).

396 Furthermore, analyzing the dynamics of dendritic spines and filopodia in living animals

397 enabled us to detect minute synaptic impairments; there are some cases in which only

398 protrusion dynamics change, without any changes in protrusion number when synaptic

399 plasticity and pathological changes are elicited (Cruz-Martin et al., 2010; Trachtenberg

400 et al., 2002). Perinatal and chronic HFD exposure leads to inflammation in the brain and 401 activation of microglia (Bilbo and Tsang, 2010; Tapia-Gonzalez et al., 2011) that

402 remove synapses (Trapp et al., 2007). Even in a resting state, microglia regulate

403 synaptic pruning during development (Paolicelli et al., 2011) and plasticity (Tremblay et

404 al., 2010). Therefore, we used the thinned-skull method allowing excitation and 
405 emission lights to penetrate the skull without inflammatory responses (Xu et al., 2007).

406

4074.2 Synaptic basis of maternal HFD-induced brain dysfunction in the adult

408 offspring

409 Previous studies have demonstrated the effects of perinatal HFD exposure on the brain

410 by evaluating molecular and behavioral alterations (Franco et al., 2012; Mischke et al.,

411 2013; Peleg-Raibstein et al., 2012; Rodriguez et al., 2012). We previously reported

412 deficits in hippocampal neurogenesis and dendritic differentiation of newborn neurons

413 in the brain of offspring exposed to HFD perinatally (Tozuka et al., 2010; Tozuka et al.,

414 2009). However, the synaptic basis of brain dysfunction in the offspring from HFD-fed

415 dams remained unclear. In the present study, we investigated the synaptic

416 endophenotype of the offspring exposed to maternal HFD to fill the gap between the

417 molecular and behavioral phenotype, and found that the offspring showed synaptic

418 instability and loss of dendritic spines. The loss of dendritic spines may be due to the

419 slightly higher elimination rate of dendritic spines than formation rate; however,

420 long-term in vivo imaging lasting for more than a week would be required to elucidate

421 the relationship between the dynamics and density of dendritic spines. The lower

422 density of spines and the higher density of filopodia suggest the deficits in synaptic

423 maturation because the ratio of filopodia to spines decreases with development

424 (Grutzendler et al., 2002).

425 Maternal HFD feeding exclusively during lactation disrupts metabolic

426 homeostasis in the offspring via impairing neuronal circuitry formation in the

427 hypothalamus (Vogt et al., 2014). HFD exposure during adolescence leads to cognitive

428 dysfunction, whereas exposure during young adulthood has no effect 
429 (Valladolid-Acebes et al., 2013). These reports and our present study suggest that the 430 synaptic developmental stage is one of the most susceptible periods to HFD toxicity 431 impairing brain function in the adult offspring. Maternal HFD exposure during 432 pregnancy confers adaptive protection against mesenteric artery dysfunction caused by 433 maternal HFD during lactation (Khan et al., 2005). This report is in consistent with our 434 result that HFD exposure exclusively during lactation induces more severe deficits than 435 HFD exposure during gestation and lactation.

436 Several lines of evidence suggest that perinatal HFD exposure causes 437 developmental delay in the brain including decreased neurogenesis, apoptosis, neuronal 438 differentiation, and behavioral deficits (Giriko et al., 2013; Niculescu and Lupu, 2009; 439 Tozuka et al., 2010; Tozuka et al., 2009). The turnover rate of dendritic spines and the 440 ratio of filopodia to total protrusions decrease with neuronal circuitry development 441 (Grutzendler et al., 2002). In a mouse model of fragile $X$ syndrome, a genetic 442 neurodevelopmental disorder, increased turnover rate and immature morphology of 443 dendritic protrusions in the mice are considered manifestations of developmental delay 444 (Cruz-Martin et al., 2010). In this context, our present findings that the offspring from 445 HFD-fed dams exhibit higher turnover rate of dendritic protrusions throughout synaptic 446 development and more filopodium density at the juvenile stage suggest that a delay in 447 neuronal circuit development may be one of the synaptic mechanisms of maternal 448 HFD-induced brain dysfunction. Increased risk of developmental disorders, such as 449 ADHD (Ray et al., 2009; Rodriguez et al., 2008) and autism spectrum disorder (Dodds 450 et al., 2011; Krakowiak et al., 2012) in children from obese mothers also supports the 451 above hypothesis.

452 Maternal obesity is associated with inflammation and excess levels of insulin 
453 and leptin, causing perturbations in the development of neural circuitry (Sullivan et al.,

454 2014), but the mechanisms for HFD programming of synaptic development and

455 behavior remain largely unknown. Our preceding study showed that maternal HFD

456 leads to peroxidized lipid accumulations in the cerebral cortex, and the product of the

457 lipid peroxidation reduces neural progenitor cell proliferation (Tozuka et al., 2009).

458 Peroxidized lipid accumulations in the cerebral cortex are reduced by the treatment of

459 ascorbic acid in drinking water (Harrison et al., 2010). Furthermore, oxidative stress

460 precedes the onset of HFD-induced obesity and insulin resistance (Matsuzawa-Nagata

461 et al., 2008). Together, the results from our previous and present studies suggest that

462 peroxidized lipid accumulation is one of the earliest steps in HFD-induced pathogenesis,

463 and exposure to oxidative stress during neuronal circuitry development may result in

464 subsequent synaptic deficits and behavioral impairments.

465

4664.3 Convergence of synapse pathology in maternal obesity and other 467 neuropsychiatric diseases: implications for comorbidity

468 Many animal models of neuropsychiatric disorders including Alzheimer's disease (Tsai 469 et al., 2004), Parkinson's disease (Villalba and Smith, 2010), vascular dementia (Sigler 470 and Murphy, 2010), autism spectrum disorder (Hatanaka et al., 2015; Hung et al., 2008; 471 Isshiki et al., 2014), schizophrenia (Fenelon et al., 2013), depression (Gourley et al., 472 2013; Liston and Gan, 2011) and anxiety disorder (Buras et al., 2013) share pathologies 473 of the synapses: protrusion instability and/or decreased mature dendritic protrusions.

474 These synaptic pathologies are also shown in our model of maternal obesity, and 475 maternal obesity is linked to an elevated risk of these neuropsychiatric disorders 476 (Sullivan et al., 2014; Van Lieshout et al., 2011). Such findings suggest that synaptic 
477 instability and immaturity may underlie the comorbidity between maternal obesity and 478 neuropsychiatric disorders. According to this commonality in the synapses, peripheral 479 pathologies used as biomarkers of perinatal HFD exposure could be applicable in the 480 diagnosis of neuropsychiatric diseases. A combined model study of maternal obesity 481 and neuropsychiatric disorders is necessary to clarify the underlying mechanisms of 482 their comorbidity. 


\section{Acknowledgements}

484 We thank Hiromi Fujita, Yoshiko Hara, Masako Shikama, Tomoko Okada and Hisae 485 Kikuchi (NCNP) for technical assistance. We are also grateful to Yoshitaka Nagai and 486 Etsuko Wada (NCNP) for informative discussion. This work was supported by a 487 Grant-in-Aid for Scientific Research on Innovative Areas "Foundation of Synapse and 488 Neurocircuit Pathology" from the Ministry of Education, Culture, Sports, Science and 489 Technology of Japan (23110527 to K.W.); and partly by Grants-in-Aid for Scientific 490 Research from the Ministry of Education, Culture, Sports, Science and Technology of 491 Japan (25290027 and 25110739 to K.W.); Intramural Research Grants for Neurological 492 and Psychiatric Disorders of NCNP (to K.W. and T.K.); a Grant-in-Aid for Young 493 Scientists (B) from the Japan Society for the Promotion of Science (25871174 to Y.H.); 494 a grant from Core Research for Evolutional Science and Technology (CREST) of the 495 Japan Science and Technology Agency (JST) (to K.W.); and a grant from Japan Agency 496 for Medical Research and Development (AMED) (to K.W.). 


\section{References}

499 Abdel-Wahab, Y.H., O'Harte, F.P., Mooney, M.H., Barnett, C.R., Flatt, P.R., 500 2002. Vitamin C supplementation decreases insulin glycation and improves

501 glucose homeostasis in obese hyperglycemic (ob/ob) mice. Metabolism 51, $502 \quad 514-517$.

503 Bilbo, S.D., Tsang, V., 2010. Enduring consequences of maternal obesity for 504 brain inflammation and behavior of offspring. FASE B J 24, 2104-2115.

505 Buras, A., Battle, L., Landers, E., Nguyen, T., Vasudevan, N., 2013. Thyroid 506 hormones regulate anxiety in the male mouse. Horm Behav 65, 88-96.

507 Cruz-Martin, A., Crespo, M., Portera-Cailliau, C., 2010. Delayed 508 stabilization of dendritic spines in fragile X mice. J Neurosci 30, 7793-7803.

509 Dodds, L., Fell, D.B., Shea, S., Armson, B.A., Allen, A.C., Bryson, S., 2011.

510 The role of prenatal, obstetric and neonatal factors in the development of

511 autism. J Autism Dev Disord 41, 891-902.

512 Elahi, M.M., Cagampang, F.R., Mukhtar, D., Anthony, F.W., Ohri, S.K.,

513 Hanson, M.A., 2009. Long-term maternal high-fat feeding from weaning

514 through pregnancy and lactation predisposes offspring to hypertension,

515 raised plasma lipids and fatty liver in mice. $\mathrm{Br}$ J Nutr 102, 514-519.

516 Fenelon, K., Xu, B., Lai, C.S., Mukai, J ., Markx, S., Stark, K.L., Hsu, P.K.,

517 Gan, W.B., Fischbach, G.D., MacDermott, A.B., Karayiorgou, M., Gogos, J .A.,

518 2013. The pattern of cortical dysfunction in a mouse model of a

519 schizophrenia-related microdeletion. J Neurosci 33, 14825-14839.

520 Ferezou-Viala, J ., Roy, A.F., Serougne, C., Gripois, D., Parquet, M., Bailleux, 
521 V., Gertler, A., Delplanque, B., Djiane, J., Riottot, M., Taouis, M., 2007.

522 Long-term consequences of maternal high-fat feeding on hypothalamic leptin

523 sensitivity and diet-induced obesity in the offspring. Am J Physiol Regul

524 Integr Comp Physiol 293, R1056-1062.

525 Franco, J.G., Fernandes, T.P., Rocha, C.P., Calvino, C., Pazos-Moura, C.C.,

526 Lisboa, P.C., Moura, E.G., Trevenzoli, I.H., 2012. Maternal high-fat diet

527 induces obesity and adrenal and thyroid dysfunction in male rat offspring at

528 weaning. J Physiol 590, 5503-5518.

529 Giriko, C.A., Andreoli, C.A., Mennitti, L.V., Hosoume, L.F., Souto Tdos, S.,

530 Silva, A.V., Mendes-da-Silva, C., 2013. Delayed physical and

531 neurobehavioral development and increased aggressive and depression-like

532 behaviors in the rat offspring of dams fed a high-fat diet. Int J Dev Neurosci

$533 \quad 31,731-739$.

534 Gourley, S.L., Swanson, A.M., Koleske, A.J ., 2013. Corticosteroid-induced

535 neural remodeling predicts behavioral vulnerability and resilience. J

536 Neurosci 33, 3107-3112.

537 Grutzendler, J., Kasthuri, N., Gan, W.B., 2002. Long-term dendritic spine 538 stability in the adult cortex. Nature 420, 812-816.

539 Harrison, F.E., Green, R.J., Dawes, S.M., May, J.M., 2010. Vitamin C

540 distribution and retention in the mouse brain. Brain Res 1348, 181-186.

541 Hatanaka, Y., Wada, K., Kabuta, T., 2015. Abnormal instability, excess

542 density, and aberrant morphology of dendritic spines in prenatally

543 testosterone-exposed mice. Neurochem Int. 
544 Huda, S.S., Brodie, L.E., Sattar, N., 2010. Obesity in pregnancy: prevalence

545 and metabolic consequences. Semin Fetal Neonatal Med 15, 70-76.

546 Hung, A.Y., Futai, K., Sala, C., Valtschanoff, J .G., Ryu, J ., Woodworth, M.A.,

547 Kidd, F.L., Sung, C.C., Miyakawa, T., Bear, M.F., Weinberg, R.J ., Sheng, M.,

548 2008. Smaller dendritic spines, weaker synaptic transmission, but enhanced

549 spatial learning in mice lacking Shank1. J Neurosci 28, 1697-1708.

550 Isshiki, M., Tanaka, S., Kuriu, T., Tabuchi, K., Takumi, T., Okabe, S., 2014.

551 Enhanced synapse remodelling as a common phenotype in mouse models of 552 autism. Nat Commun 5, 4742.

553 Kanagalingam, M.G., Forouhi, N.G., Greer, I.A., Sattar, N., 2005. Changes in

554 booking body mass index over a decade: retrospective analysis from a

555 Glasgow Maternity Hospital. BJ OG 112, 1431-1433.

556 Khan, I.Y., Dekou, V., Douglas, G., J ensen, R., Hanson, M.A., Poston, L., 557 Taylor, P.D., 2005. A high-fat diet during rat pregnancy or suckling induces 558 cardiovascular dysfunction in adult offspring. Am J Physiol Regul Integr 559 Comp Physiol 288, R127-133.

560 Kopel man, P.G., 2000. Obesity as a medical problem. Nature 404, 635-643.

561 Krakowiak, P., Walker, C.K., Bremer, A.A., Baker, A.S., Ozonoff, S., Hansen, 562 R.L., Hertz-Picciotto, I., 2012. Maternal metabolic conditions and risk for 563 autism and other neurodevel opmental disorders. Pediatrics 129, el121-1128. 564 Liston, C., Gan, W.B., 2011. Glucocorticoids are critical regulators of 565 dendritic spine development and plasticity in vivo. Proc Natl Acad Sci U S A $566108,16074-16079$. 
567 Matsuzawa-Nagata, N., Takamura, T., Ando, H., Nakamura, S., Kurita, S.,

568 Misu, H., Ota, T., Yokoyama, M., Honda, M., Miyamoto, K., Kaneko, S., 2008.

569 Increased oxidative stress precedes the onset of high-fat diet-induced insulin

570 resistance and obesity. Metabolism 57, 1071-1077.

571 Mischke, M., Pruis, M.G., Boekschoten, M.V., Groen, A.K., Fitri, A.R., van de

572 Heijning, B.J ., Verkade, H.J ., Muller, M., Plosch, T., Steegenga, W.T., 2013.

573 Maternal Western-style high fat diet induces sex-specific physiological and

574 molecular changes in two-week-old mouse offspring. PLoS One 8, e78623.

575 Niculescu, M.D., Lupu, D.S., 2009. High fat diet-induced maternal obesity

576 alters fetal hippocampal development. Int J Dev Neurosci 27, 627-633.

577 Oike, Y., Akao, M., Yasunaga, K., Yamauchi, T., Morisada, T., Ito, Y., U rano, 578 T., Kimura, Y., Kubota, Y., Maekawa, H., Miyamoto, T., Miyata, K., 579 Matsumoto, S., Sakai, J ., Nakagata, N., Takeya, M., Koseki, H., Ogawa, Y., 580 Kadowaki, T., Suda, T., 2005. Angiopoi etin-related growth factor antagonizes 581 obesity and insulin resistance. Nat Med 11, 400-408.

582 Paolicelli, R.C., Bolasco, G., Pagani, F., Maggi, L., Scianni, M., Panzanelli, P., 583 Giustetto, M., Ferreira, T.A., Guiducci, E., Dumas, L., Ragozzino, D., Gross, 584 C.T., 2011. Synaptic pruning by microglia is necessary for normal brain 585 development. Science 333, 1456-1458.

586 Peleg-Raibstein, D., Luca, E., Wolfrum, C., 2012. Maternal high-fat diet in 587 mice programs emotional behavior in adulthood. Behav Brain Res 233, $588 \quad 398-404$.

589 Penzes, P., Cahill, M.E., J ones, K.A., VanLeeuwen, J .E., Woolfrey, K.M., 2011. 
590 Dendritic spine pathology in neuropsychiatric disorders. Nat Neurosci 14, $591 \quad 285-293$.

592 Ray, G.T., Croen, L.A., Habel, L.A., 2009. Mothers of children diagnosed with 593 attention-deficit/hyperactivity disorder: health conditions and medical care 594 utilization in periods before and after birth of the child. Med Care 47, $595 \quad 105-114$.

596 Rodriguez, A., Miettunen, J ., Henriksen, T.B., Olsen, J ., Obel, C., Taanila, A., 597 Ebeling, H., Linnet, K.M., Moilanen, I., J arvelin, M.R., 2008. Maternal 598 adiposity prior to pregnancy is associated with ADHD symptoms in 599 offspring: evidence from three prospective pregnancy cohorts. Int J Obes 600 (Lond) 32, 550-557.

601 Rodriguez, J.S., Rodriguez-Gonzalez, G.L., Reyes-Castro, L.A., I banez, C., 602 Ramirez, A., Chavira, R., Larrea, F., Nathanielsz, P.W., Zambrano, E., 2012. 603 Maternal obesity in the rat programs male offspring exploratory, learning 604 and motivation behavior: prevention by dietary intervention pre-gestation or 605 in gestation. Int J Dev Neurosci 30, 75-81.

606 Samuelsson, A.M., Matthews, P.A., Argenton, M., Christie, M.R., McConnell, 607 J .M., J ansen, E.H., Piersma, A.H., Ozanne, S.E., Twinn, D.F., Remacle, C., 608 Rowlerson, A., Poston, L., Taylor, P.D., 2008. Diet-induced obesity in female 609 mice leads to offspring hyperphagia, adiposity, hypertension, and insulin 610 resistance: a novel murine model of developmental programming. 611 Hypertension 51, 383-392.

612 Sigler, A., Murphy, T.H., 2010. In vivo 2-photon imaging of fine structure in 
613 the rodent brain: before, during, and after stroke. Stroke 41, S117-123.

614 Souza, C.G., Moreira, J .D., Siqueira, I.R., Pereira, A.G., Rieger, D.K., Souza, 615 D.O., Souza, T.M., Portela, L.V., Perry, M.L., 2007. Highly palatable diet 616 consumption increases protein oxidation in rat frontal cortex and 617 anxiety-like behavior. Life Sci 81, 198-203.

618 Stranahan, A.M., Norman, E.D., Lee, K., Cutler, R.G., Telljohann, R.S., Egan, 619 J.M., Mattson, M.P., 2008. Diet-induced insulin resistance impairs 620 hippocampal synaptic plasticity and cognition in middle-aged rats. 621 Hippocampus 18, 1085-1088.

622 Sullivan, E.L., Nousen, E.K., Chamlou, K.A., 2014. Maternal high fat diet 623 consumption during the perinatal period programs offspring behavior. 624 Physiol Behav 123, 236-242.

625 Tapia-Gonzalez, S., Garcia-Segura, L.M., Tena-Sempere, M., Frago, L.M., 626 Castellano, J .M., Fuente-Martin, E., Garcia-Caceres, C., Argente, J ., Chowen, 627 J.A., 2011. Activation of microglia in specific hypothalamic nuclei and the 628 cerebellum of adult rats exposed to neonatal overnutrition. J 629 Neuroendocrinol 23, 365-370.

630 Tozuka, Y., Kumon, M., Wada, E., Onodera, M., Mochizuki, H., Wada, K., 631 2010. Maternal obesity impairs hippocampal BDNF production and spatial 632 learning performance in young mouse offspring. Neurochem Int 57, 235-247. 633 Tozuka, Y., Wada, E., Wada, K., 2009. Diet-induced obesity in female mice 634 leads to peroxidized lipid accumulations and impairment of hippocampal 635 neurogenesis during the early life of their offspring. FASEB J 23, 1920-1934. 
636 Trachtenberg, J .T., Chen, B.E., Knott, G.W., Feng, G., Sanes, J .R., Welker, E., 637 Svoboda, K., 2002. Long-term in vivo imaging of experience-dependent 638 synaptic plasticity in adult cortex. Nature 420, 788-794.

639 Trapp, B.D., Wujek, J .R., Criste, G.A., J alabi, W., Yin, X., Kidd, G.J ., 640 Stohlman, S., Ransohoff, R., 2007. Evidence for synaptic stripping by cortical 641 microglia. Glia 55, 360-368.

642 Tremblay, M.E., Lowery, R.L., Majewska, A.K., 2010. Microglial interactions 643 with synapses are modulated by visual experience. PLoS Biol 8, e1000527.

644 Tsai, J., Grutzendler, J., Duff, K., Gan, W.B., 2004. Fibrillar amyloid 645 deposition leads to local synaptic abnormalities and breakage of neuronal 646 branches. Nat Neurosci 7, 1181-1183.

647 Valladolid-Acebes, I., Fole, A., Martin, M., Morales, L., Cano, M.V., 648 Ruiz-Gayo, M., Del Olmo, N., 2013. Spatial memory impairment and changes 649 in hippocampal morphology are triggered by high-fat diets in adolescent 650 mice. Is there a role of leptin? Neurobiol Learn Mem 106, 18-25.

651 Van Lieshout, R.J., Taylor, V.H., Boyle, M.H., 2011. Prepregnancy and 652 pregnancy obesity and neurodevelopmental outcomes in offspring: a 653 systematic review. Obes Rev 12, e548-559.

654 Villalba, R.M., Smith, Y., 2010. Striatal spine plasticity in Parkinson's 655 disease. Front Neuroanat 4, 133.

656 Vogt, M.C., Paeger, L., Hess, S., Steculorum, S.M., Awazawa, M., Hampel, B., 657 Neupert, S., Nicholls, H.T., Mauer, J ., Hausen, A.C., Predel, R., Kloppenburg, 658 P., Horvath, T.L., Bruning, J.C., 2014. Neonatal insulin action impairs 
659 hypothalamic neurocircuit formation in response to maternal high-fat 660 feeding. Cell 156, 495-509.

661 Williams, L., Seki, Y., Vuguin, P.M., Charron, M.J ., 2013. Animal models of in 662 utero exposure to a high fat diet: A review. Biochim Biophys Acta.

663 Wu, A., Ying, Z., Gomez-Pinilla, F., 2004. The interplay between oxidative 664 stress and brain-derived neurotrophic factor modulates the outcome of a 665 saturated fat diet on synaptic plasticity and cognition. Eur J Neurosci 19, $666 \quad 1699-1707$.

667 Wu, T., Deng, S., Li, W.G., Yu, Y., Li, F., Mao, M., 2013. Maternal obesity 668 caused by overnutrition exposure leads to reversal learning deficits and 669 striatal disturbance in rats. PLoS One 8, e78876.

670 Xu, H.T., Pan, F., Yang, G., Gan, W.B., 2007. Choice of cranial window type 671 for in vivo imaging affects dendritic spine turnover in the cortex. Nat 672 Neurosci 10, 549-551.

673 Yang, G., Pan, F., Parkhurst, C.N., Grutzendler, J., Gan, W.B., 2010. 674 Thinned-skull cranial window technique for long-term imaging of the cortex 675 in live mice. Nat Protoc 5, 201-208.

676 Zhang, X., Dong, F., Ren, J ., Driscoll, M.J ., Culver, B., 2005. High dietary fat 677 induces NADPH oxidase-associated oxidative stress and inflammation in rat 678 cerebral cortex. Exp Neurol 191, 318-325. 679 


\section{Legends}

681 Figure 1. Maternal HFD leads to instability of dendritic spines and filopodia in the 682 adult offspring. a, Schematic diagram of maternal and offspring diet protocols. In the 683 ND/ND group, dams were fed ND throughout gestation and lactation. In the HFD/ND 684 group, dams were fed HFD throughout gestation and lactation. In both groups, dams 685 and pups were fed ND from lactational day 16. b, In vivo two-photon time-lapse 686 imaging of L1 dendrites from L5 pyramidal neurons in 10-week-old ND/ND $(n=11$ 687 dendrites from four mice) and HFD/ND $(n=21$ dendrites from seven mice) mice. 688 Repeated images of the identical dendrites over $1 \mathrm{~h}$ in each group show increased 689 formation (filled arrowheads) and elimination (open arrowheads) of dendritic spines 690 (filled arrow) and filopodia (open arrow). Images are best projections (3-7 optical 691 sections, $0.75 \mu \mathrm{m}$ apart). c, Percentages of spines formed or eliminated as a proportion 692 of total spines. HFD/ND mice showed a greater elimination rate of spines. d, 693 Percentages of filopodia formed or eliminated as a proportion of total filopodia. Both 694 formation and elimination rate were greater in HFD/ND mice. Note that both the 695 formation and elimination rates of filopodia are much higher than those of spines in the 696 control (ND/ND) group. Data are presented as mean \pm SEM. ${ }^{* *} p<0.01$, Student's $697 t$-test. Scale bar, $5 \mu \mathrm{m}$.

699 Figure 2. Maternal HFD-exposed offspring develop synaptic instability and loss of 700 dendritic spines during synaptic maturation. a, Turnover rates of spines (ratio of spines 701 formed and eliminated to twice the total number of spines) at 4 weeks (ND/ND: $n=13$ 702 dendrites from five mice; HFD/ND: $n=18$ dendrites from five mice), 6 weeks

703 (ND/ND: $n=12$ dendrites from four mice; HFD/ND: $n=18$ dendrites from five mice), 
7048 weeks (ND/ND: $n=15$ dendrites from five mice; HFD/ND: $n=18$ dendrites from

705 seven mice) and 10 weeks of age. HFD/ND mice showed higher spine turnover rates

706 than ND/ND mice throughout maturation. $\mathbf{b}$, Turnover rates of filopodia at $4,6,8$ and

70710 weeks of ages. HFD/ND mice showed higher filopodium turnover rates than ND/ND

708 mice from 6 weeks of age. c, The density of dendritic protrusions including both spines

709 and filopodia, spines and filopodia in ND/ND and HFD/ND mice at each age. In

$710 \mathrm{HFD} / \mathrm{ND}$ mice, the loss of dendritic spines was developed with aging whereas

711 filopodium density was slightly higher at each age. Data are presented as mean $\pm \mathrm{SEM}$.

$712 * p<0.05, * * p<0.01, * * * p<0.001$, two-way ANOVA followed by Bonferroni's test

713 (a, b) or Student's t-test (c).

714

715 Figure 3. HFD exposure both before and after weaning causes significant loss of

716 dendritic spines. a, Experimental paradigm for maternal and offspring diets. In the

717 ND/ND group, both dams and pups were fed ND until imaging. In the HFD/ND group,

718 dams were fed HFD during gestation and lactation, and pups were fed ND after weaning.

719 In the ND/HFD group, dams were fed ND, and pups were fed HFD after weaning. In

720 the HFD/HFD group, both dams and pups were fed HFD. b, In vivo two-photon

721 imaging over $1 \mathrm{~h}$ of L1 dendrites from L5 pyramidal neurons in 8-week-old ND/ND,

722 HFD/ND, HFD/ND ( $\mathrm{n}=16$ dendrites from four mice) and HFD/HFD $(n=12$ dendrites

723 from four mice) mice. Repeated images of the identical dendrites over $1 \mathrm{~h}$ in each group

724 show a higher rate of formation (filled arrowheads) and elimination (open arrowheads)

725 of dendritic spines and filopodia in HFD/ND, ND/HFD and HFD/HFD groups. Images

726 are best projections (3-7 optical sections, $0.75 \mu \mathrm{m}$ apart). c, Percentages of spines

727 formed or eliminated as a proportion of total spines. HFD/HFD mice exhibited greater 
728 spine formation and elimination rates than ND/ND mice. d, Percentages of filopodia 729 formed or eliminated as a proportion of total filopodia. HFD/HFD mice showed greater 730 rates of filopodium formation and elimination than ND/ND mice, but there were no

731 significant differences in spine and filopodium dynamics among HFD/ND, ND/HFD 732 and HFD/HFD groups. e, Dendritic protrusion density in ND/ND, HFD/ND, ND/HFD 733 and HFD/HFD mice. HFD/HFD mice showed a lower total protrusion density as a 734 result of having significantly fewer spines. HFD/HFD mice had fewer spines than both 735 HFD/ND and ND/HFD mice. Data are presented as mean \pm SEM. ${ }^{* *} p<0.01, * * * p<$ 7360.001 (compared with ND/ND), $\uparrow p<0.05, \dagger \dagger p<0.01$ (compared with HFD/ND), $\$ p$

$737<0.05,+4 p<0.001$ (compared with ND/HFD), one-way ANOVA followed by 738 Tukey's test. Scale bar, $5 \mu \mathrm{m}$.

739

740 Figure 4. Maternal HFD exclusively during lactational stage leads to synaptic 741 instability and loss of dendritic spines. a, Schematic diagram of maternal and offspring 742 diets. In the $\mathrm{HFD}_{\text {lad }}$ ND group, dams were fed HFD exclusively during lactation, and 743 pups were fed ND after weaning. b, In vivo two-photon imaging over $1 \mathrm{~h}$ of an identical

744 L1 dendrite from L5 pyramidal neurons in 8-week-old $\mathrm{HFD}_{\text {lac }} / \mathrm{ND}$ group $(n=15$ 745 dendrites from seven mice). Formation (filled arrowheads) and elimination (open 746 arrowhead) of dendritic spines and filopodia are indicated. Images are best projections 747 (3-7 optical sections, $0.75 \mu \mathrm{m}$ apart). c, Percentages of spines formed or eliminated as a 748 proportion of total spines in ND/ND, HFD/ND and $\mathrm{HFD}_{\mathrm{lac}} / \mathrm{HFD}$ mice. $\mathrm{HFD}_{\mathrm{lac}} / \mathrm{ND}$ mice 749 exhibited greater spine formation and elimination rates than ND/ND mice. d, 750 Percentages of filopodia formed or eliminated as a proportion of total filopodia. $751 \mathrm{HFD}_{\text {lac }} \mathrm{ND}$ mice showed higher rates of filopodium formation and elimination than 
752 ND/ND mice. e, Dendritic protrusion density. $\mathrm{HFD}_{\text {lad }}$ ND mice showed a lower spine 753 density than ND/ND mice. Data are presented as mean \pm SEM. $* p<0.05, * * p<0.01$, $754 * * * p<0.001$ (compared with ND/ND), $\uparrow p<0.05$ (compared with HFD/ND), one-way 755 ANOVA followed by Tukey's test. Scale bar, $5 \mu \mathrm{m}$.

756

757 Figure 5. Antioxidant intake during lactation ameliorates the synaptic instability 758 induced by maternal HFD in the adult offspring. a, Schematic diagram of maternal and 759 offspring diets. In the $\mathrm{ND}_{\mathrm{AA}} / \mathrm{ND}$ group, dams were fed ND with administration of 760 ascorbic acid in drinking water during lactation, and pups were fed ND after weaning. 761 In the $\mathrm{HFD}_{\mathrm{AA}} / \mathrm{ND}$ group, dams were fed HFD throughout gestation and lactation with 762 administration of ascorbic acid during lactation, and pups were fed ND after weaning. b, 763 In vivo two-photon imaging over $1 \mathrm{~h}$ of identical L1 dendrites from L5 pyramidal 764 neurons in 10 -week-old $\mathrm{ND}_{\mathrm{AA}} / \mathrm{ND}(n=10$ dendrites from six mice $)$ and $\mathrm{HFD}_{\mathrm{AA}} / \mathrm{ND}(n$ $765=10$ dendrites from four mice) mice. Formation (filled arrowheads) and elimination 766 (open arrowhead) of dendritic spines and filopodia are indicated. Images are best 767 projections (3-7 optical sections, $0.75 \mu \mathrm{m}$ apart). c, Percentages of spines formed or 768 eliminated as a proportion of total spines in $\mathrm{ND} / \mathrm{ND}, \mathrm{HFD} / \mathrm{ND}, \mathrm{ND}_{\mathrm{AA}} / \mathrm{ND}$ and $769 \mathrm{HFD}_{\mathrm{AA}} / \mathrm{ND}$ mice. $\mathrm{HFD}_{\mathrm{AA}} / \mathrm{ND}$ mice showed lower spine formation and elimination rates 770 than HFD/ND mice. d, Percentages of filopodia formed or eliminated as a proportion of 771 total filopodia. $\mathrm{HFD}_{\mathrm{AA}} / \mathrm{ND}$ mice showed slightly lower rates of filopodium formation 772 and elimination than HFD/ND mice. e, Dendritic protrusion density. $\mathrm{HFD}_{\mathrm{AA}} / \mathrm{ND}$ mice 773 exhibited slightly higher density of dendritic spines than HFD/ND mice, but there was 774 no significant difference between the two groups. Data are presented as mean $\pm \mathrm{SEM}$. $775 * * p<0.01, * * * p<0.001$ (compared with ND/ND), $\dagger p<0.05, \dagger \dagger p<0.01, \dagger \dagger \dagger p<$ 
7760.001 (compared with HFD/ND), one-way ANOVA followed by Tukey's test. Scale bar, $7775 \mu \mathrm{m}$. 
Figure 1.

a

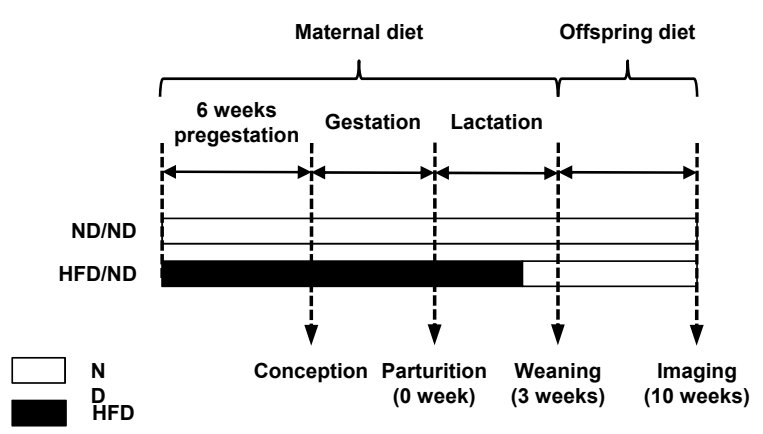

C

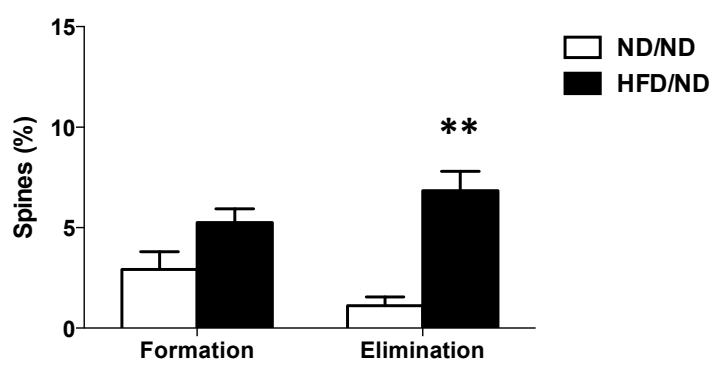

b

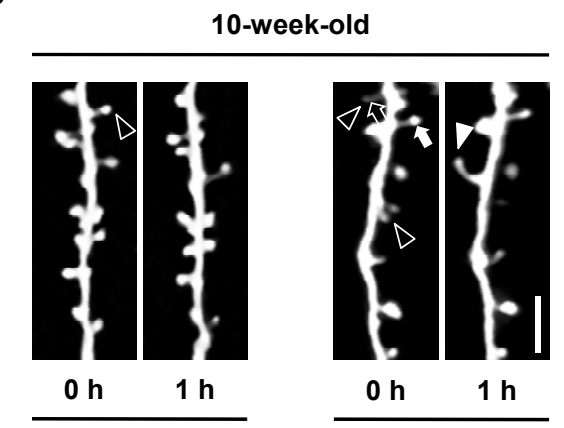

ND/ND

HFD/ND

d

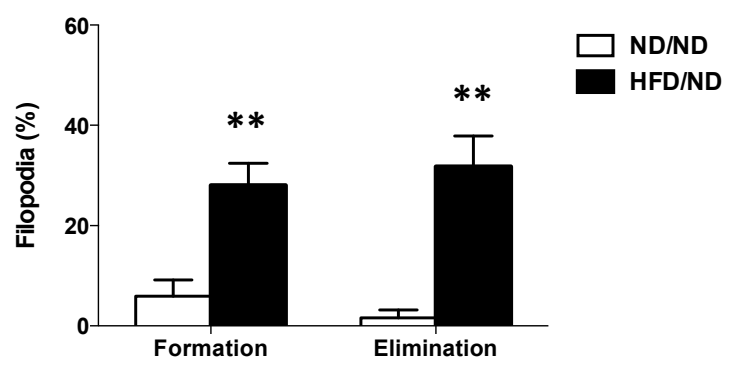


Figure 2.

a

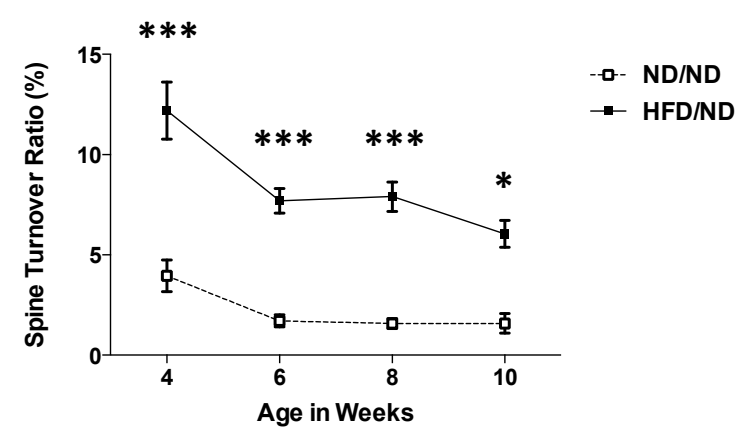

c
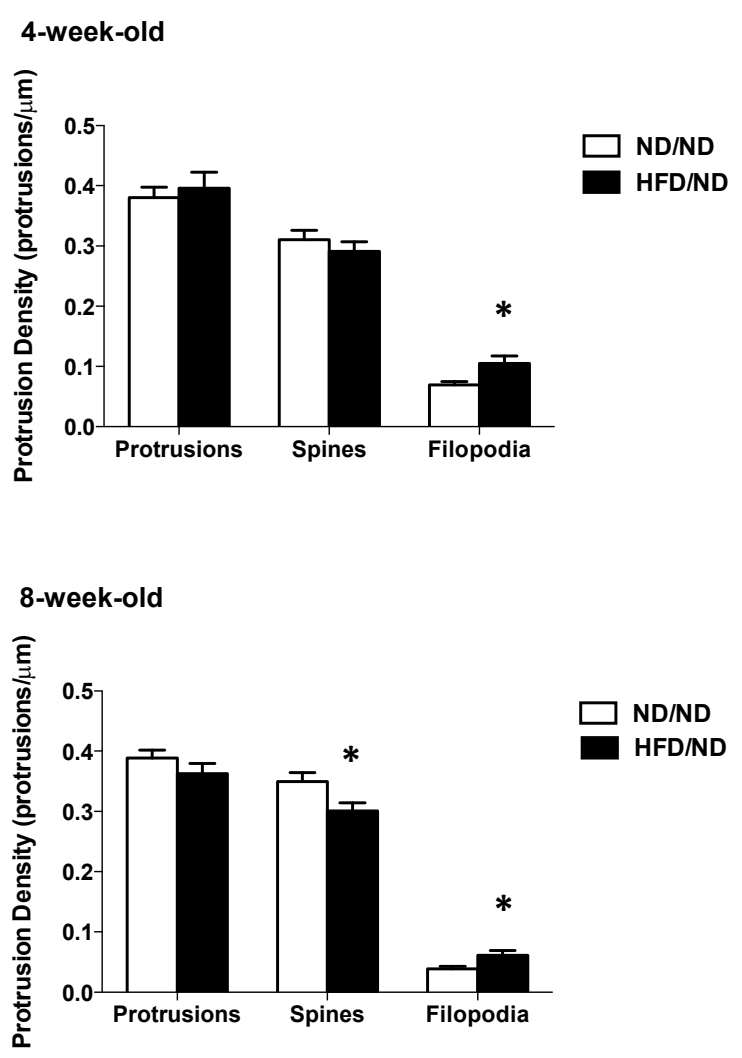

b
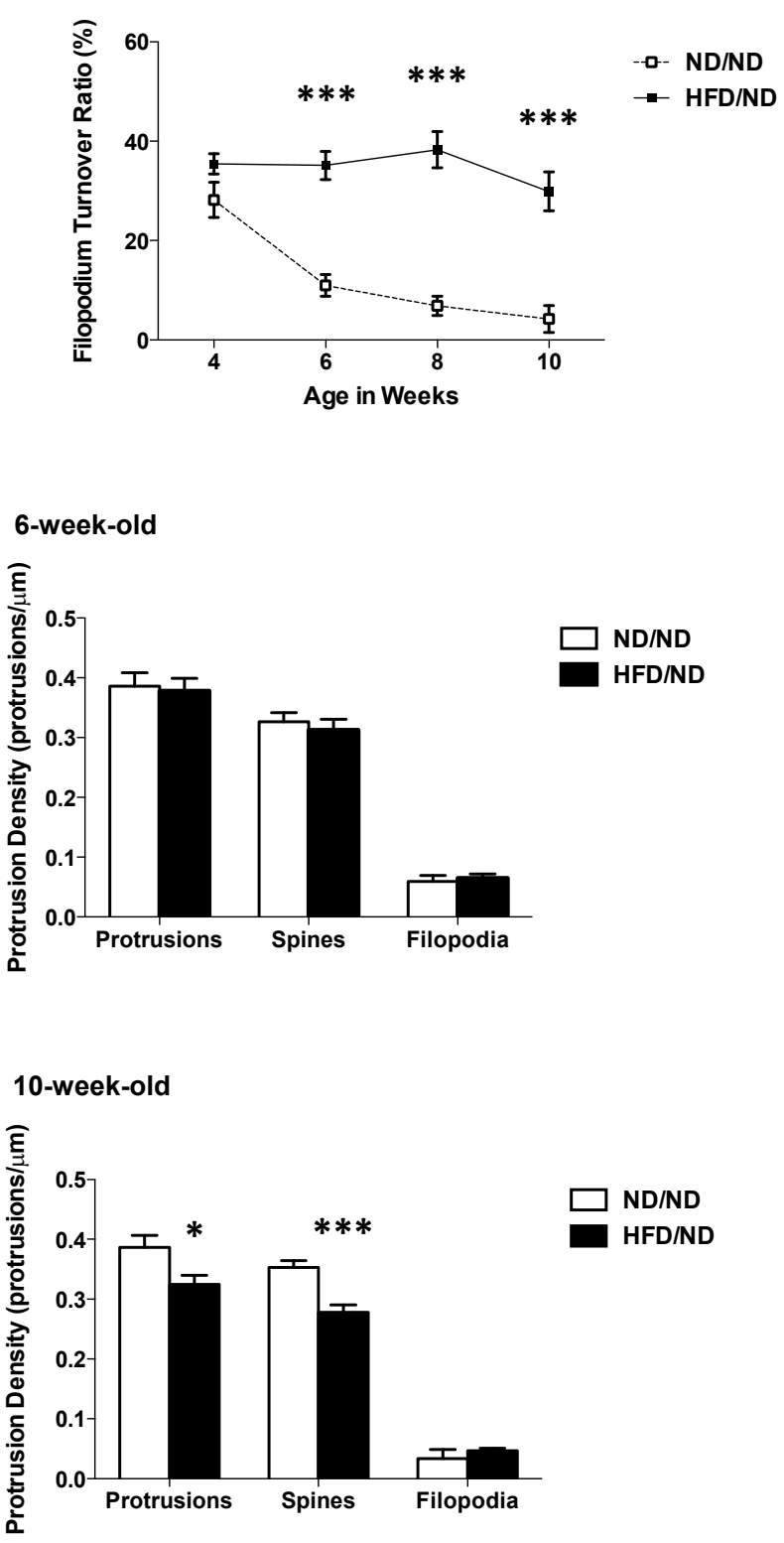
Figure 3.

a

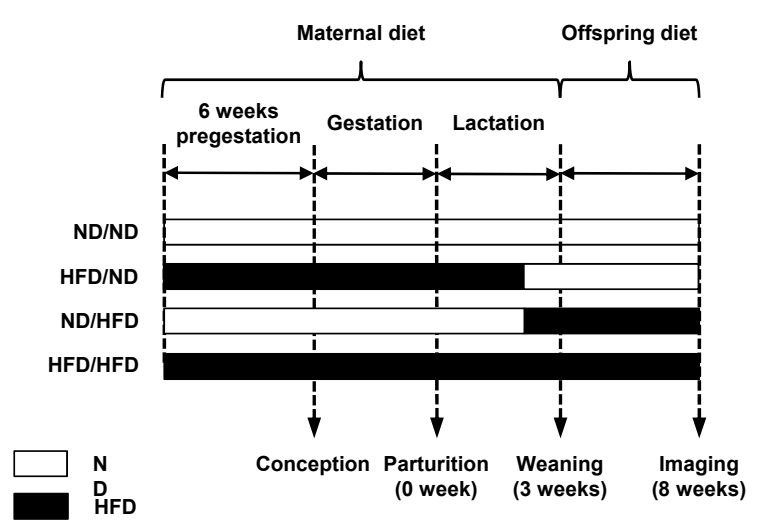

C

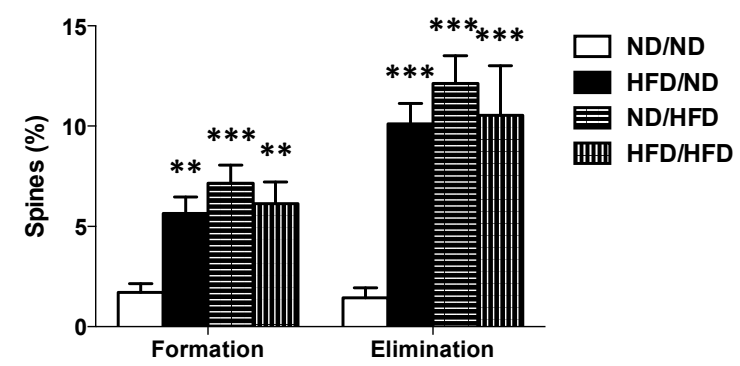

e

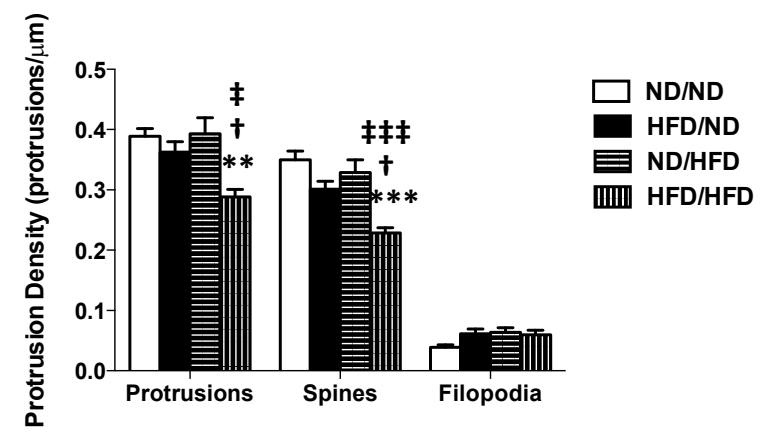

b

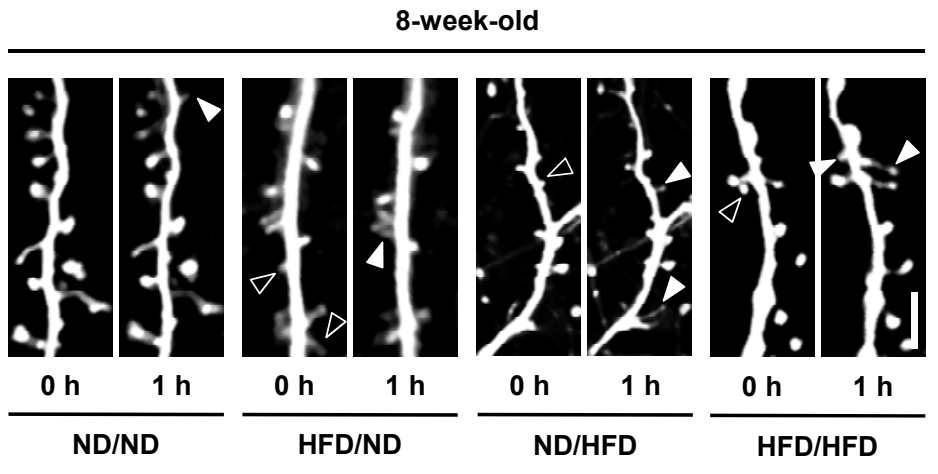

d

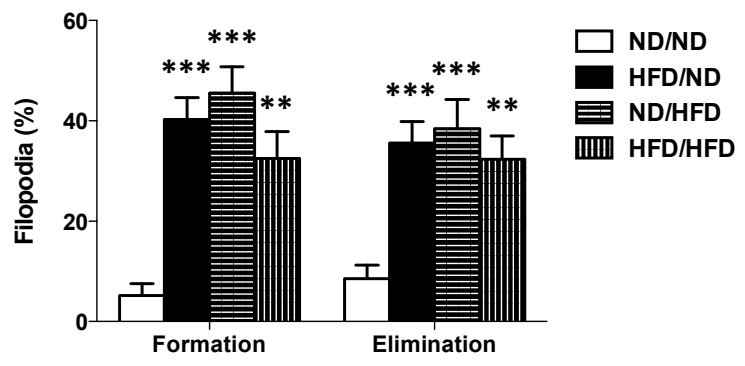


Figure 4.

a

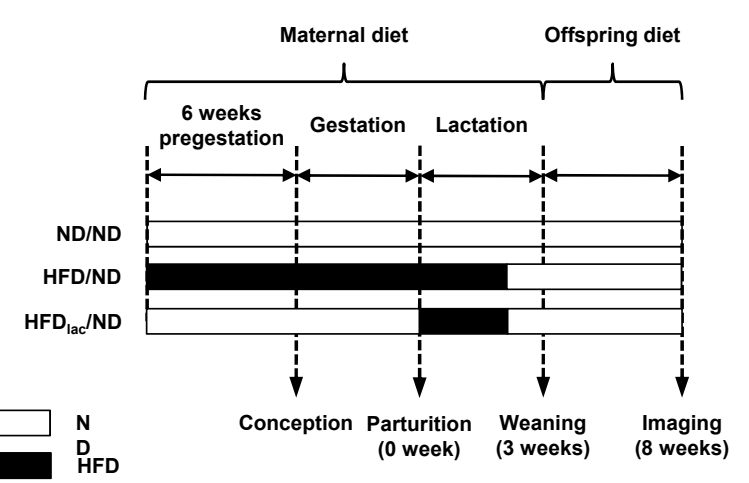

C

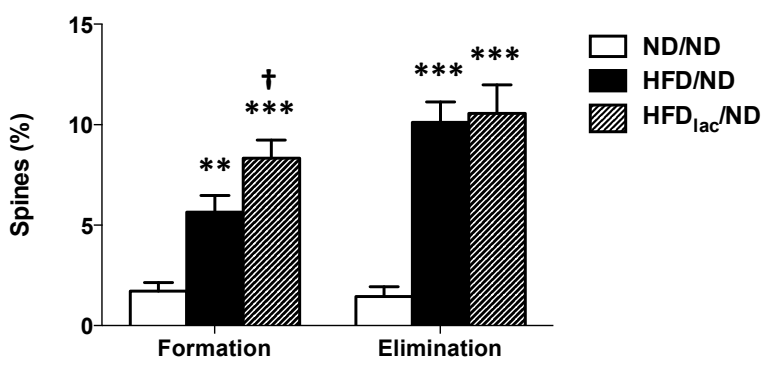

e

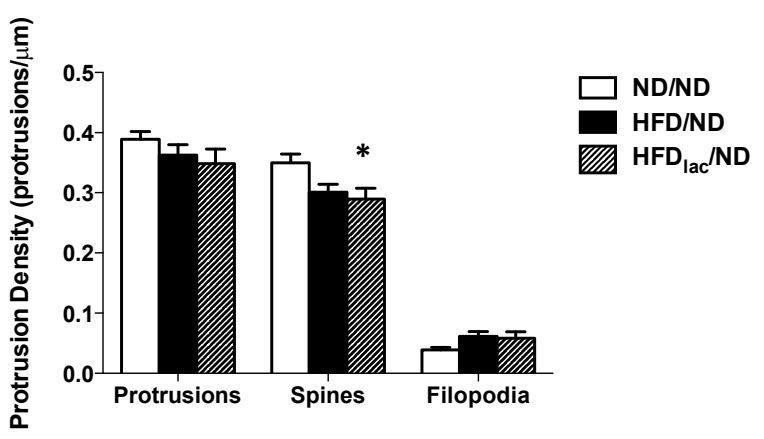

b

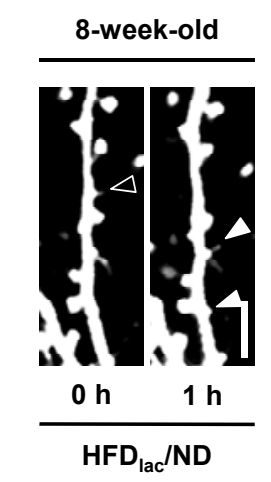

d

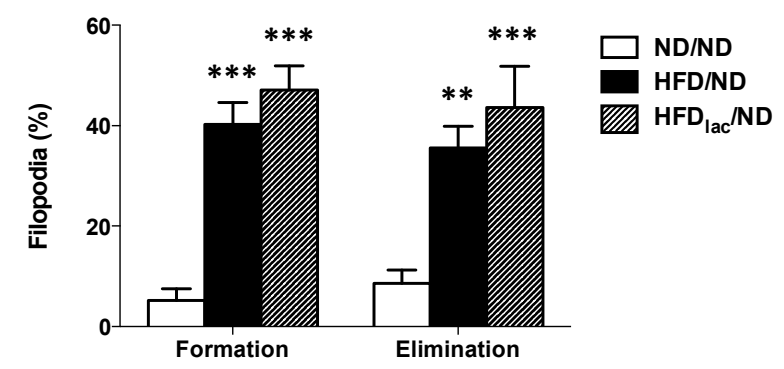


Figure 5.

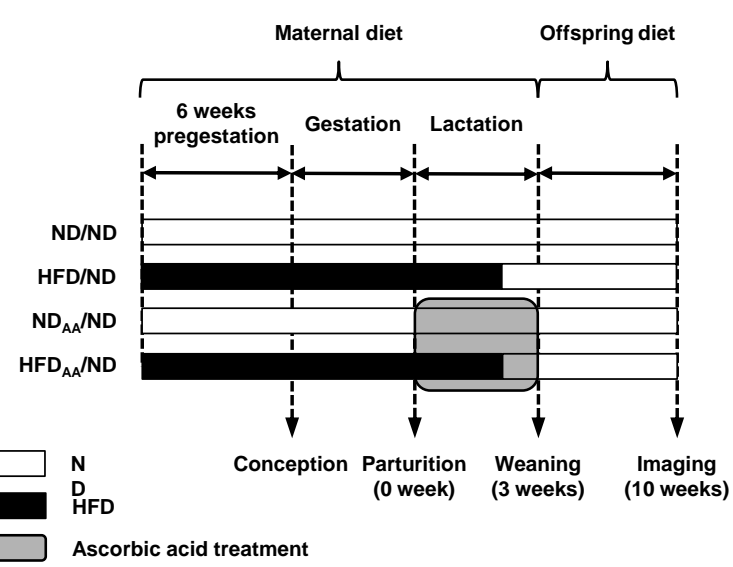

c

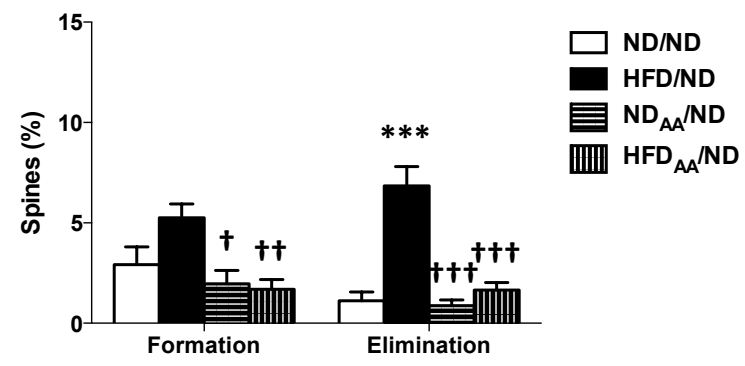

e

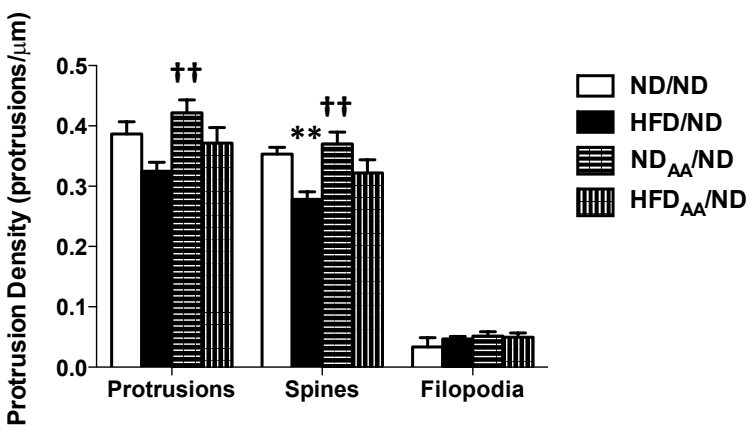

b

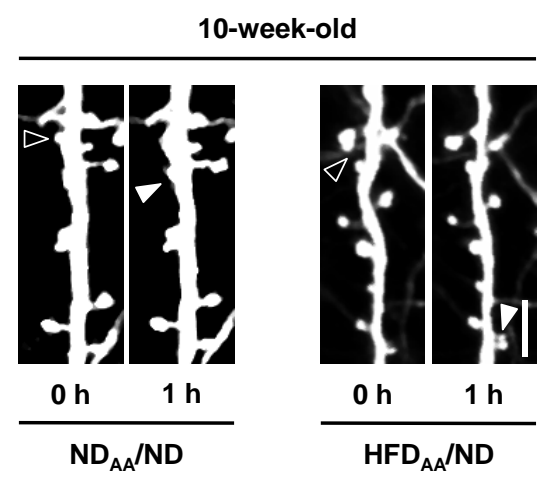

d

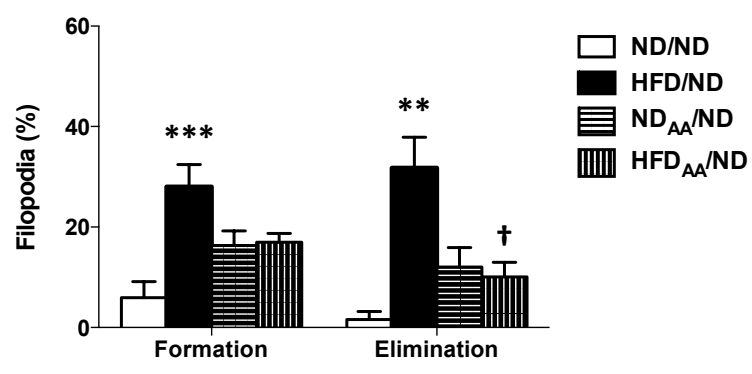

